\title{
UNBOUNDED OPERATORS, LIE ALGEBRAS, AND LOCAL REPRESENTATIONS
}

\author{
PALLE JORGENSEN AND FENG TIAN
}

\begin{abstract}
We prove a number of results on integrability and extendability of Lie algebras of unbounded skew-symmetric operators with common dense domain in Hilbert space. By integrability for a Lie algebra $\mathfrak{g}$, we mean that there is an associated unitary representation $\mathcal{U}$ of the corresponding simply connected Lie group such that $\mathfrak{g}$ is the differential of $\mathcal{U}$. Our results extend earlier integrability results in the literature; and are new even in the case of a single operator. Our applications include a new invariant for certain Riemann surfaces.
\end{abstract}

\section{Contents}

1. Introduction 1

2. One dimension, single operators 2

3. Commuting skew-symmetric operators with common dense domain 5

4. Lie algebras, and local representations of Lie groups 7

$\begin{array}{ll}\text { Appendix. Riemann surfaces of finite-cover degree } & 16\end{array}$

$\begin{array}{ll}\text { References } & 18\end{array}$

\section{INTRODUCTION}

In this paper we discuss the problem of integrating representations of Lie algebras to unitary representations of the corresponding simply connected Lie group. Our main purpose is to stress a link between the two, taking the form of local representations. Hence we begin with the case of one dimension, so the real line $\mathbb{R}$, in section 2 , and we turn to general Lie algebras/Lie groups in section 4 . The literature is vast, and to give the reader a sense of different directions, both current and classical, we suggest the following papers, and the sources cited there: [Jor87, Jor86, FS66, Seg64, Ben02, Pra91, Voh72, Che72, Arn78, dG84, dG83, Nee11, GKS11, GT12, Nee06, Rob90, Rob89,

2000 Mathematics Subject Classification. Primary 47L60, 46N30, 46N50, 42C15, 65R10, 05C50, 05C75, 31C20; Secondary 46N20, 22E70, 31A15, 58J65, 81S25.

Key words and phrases. Unbounded operators, deficiency-indices, unbounded operators, extensions, Hilbert space, boundary values, non-commutative geometry, Lie algebras, Lie groups, unitary representations, locally invariant domain, Riemann surface, harmonic analysis, Hilbert space. 
BGJR88, Rus87, Frö80, Nel59]. Applications are diverse as well; physics (symmetry groups, relativistic and non-relativistic), differential equations, harmonic analysis, and stochastic processes.

The simplest case of the integrability problem for Lie algebras of unbounded operators is that of a single operator. Here we will restrict attention to skew-symmetric operators with dense domain in Hilbert space $\mathscr{H}$. The story begins with von Neumann's theory of indices, also called defect indices (or deficiency indices); so named because they measure the gap between an operator being formally skew-adjoint on the one hand, and skew-adjoint on the other. By the latter, we mean that it has a spectral resolution, and therefore is the generator of a strongly continuous one-parameter group of unitary operators in $\mathscr{H}$. This is really a geometric formulation of a variety of boundary value problems.

The paper is organized as follows: In section 2 we present the case of a single operator. This will be used, and it also allows us to introduce key ideas to be used later, for abelian Lie algebras in section 3, in the case of non-abelian Lie algebras of unbounded operators in section 4 .

\section{ONE DIMENSION, SINGLE OPERATORS}

In the case of a single skew-symmetric operator $T$ with dense domain in a Hilbert space $\mathscr{H}$, we introduce a notion of "local invariance," and we prove that it is equivalent to $T$ having von Neumann indices $(0,0)$, i.e., to $T$ being essentially skew-adjoint. Equivalently, indices $(0,0)$ means that $T$ has a projection valued spectral resolution; and is therefore the infinitesimal generator of a strongly continuous one-parameter group of unitary operators in $\mathscr{H}$.

The systematic study of extensions of symmetric (or equivalently skew-symmetric) operators began with von Neumann's paper [vN30]. Different applications, including boundary value problems and scattering theory, see [DS88] and [LP89]. For more recent applications, see for example [JPT14, JPT13, AJSV13, CJ12].

Theorem 2.1. Let $H$ be a skew-symmetric operator with dense domain $\mathscr{D}$ in a Hilbert space $\mathscr{H}$, i.e.,

$$
\langle H v, w\rangle+\langle v, H w\rangle=0
$$

for all $v, w \in \mathscr{D}$. Suppose there are subspaces $\mathscr{D}_{\varepsilon}, \varepsilon \in \mathbb{R}_{+}$, such that

(i)

$$
\mathscr{D}=\bigcup_{\varepsilon \in \mathbb{R}_{+}} \mathscr{D}_{\varepsilon} . \text { (Note that } \mathscr{D}_{\varepsilon} \text { may be zero if } \varepsilon \text { is large.) }
$$

(ii) For every $\varepsilon \in \mathbb{R}_{+}$there is an $\varepsilon^{\prime}, 0<\varepsilon^{\prime}<\varepsilon$, and there are operators

$$
\left\{\varphi_{\varepsilon}(t):|t|<\varepsilon\right\}
$$

with dense domain $\mathscr{D}$ such that:

(a) $\varphi_{\varepsilon}(s+t)=\varphi_{\varepsilon}(s) \varphi_{\varepsilon}(t),|s|<\varepsilon,|t|<\varepsilon,|s+t|<\varepsilon$;

(b) $\frac{d}{d t} \varphi_{\varepsilon}(t)=H \varphi_{\varepsilon}(t),|t|<\varepsilon^{\prime}, \varphi_{\varepsilon}(0)=I$; and 
(c) $\varphi_{\varepsilon}(t)$ leaves $\mathscr{D}_{\varepsilon}$ invariant for all $t \in\left(-\varepsilon^{\prime}, \varepsilon^{\prime}\right)$.

Then the operator $H$ is essentially skew-adjoint, i.e., it has a projection-valued spectral resolution.

Proof. Step 1 For all $\varepsilon \in \mathbb{R}_{+}$, and $v \in \mathscr{D}_{\varepsilon}$, we have

$$
\left\|\varphi_{\varepsilon}(t) v\right\|=\|v\|, \forall t \in(-\varepsilon, \varepsilon) .
$$

Indeed, let $v \in \mathscr{D}_{\varepsilon}$ be as above; then

$$
\begin{aligned}
& \frac{d}{d t}\left\|\varphi_{\varepsilon}(t) v\right\|^{2} \quad=\left\langle\frac{d}{d t} \varphi_{\varepsilon}(t) v, \varphi_{\varepsilon}(t) v\right\rangle+\left\langle\varphi_{\varepsilon}(t) v, \frac{d}{d t} \varphi_{\varepsilon}(t) v\right\rangle
\end{aligned}
$$

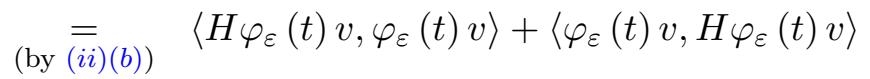

$$
\begin{aligned}
& \text { (by } \overline{(2.1))}
\end{aligned}
$$

Hence (2.2) follows.

Step 2 We get a strongly continuous unitary one-parameter group $\left\{U_{t} \mid t \in \mathbb{R}\right\}$ acting in $\mathscr{H}$, by extending from the local flows $\left\{\varphi_{\varepsilon}(t)|| t \mid<\varepsilon\right\}$, as follows:

Pick $t \in \mathbb{R} \backslash\{0\}$, and $n \in \mathbb{N}$ s.t. $|t / n|<\varepsilon^{\prime}$, where $\varepsilon^{\prime}$ is as in (ii). Then the operators $\varphi_{\varepsilon}(t / n)$ leave invariant the fixed subspaces $\mathscr{D}_{\varepsilon}$ from (i). For $v \in \mathscr{D}_{\varepsilon}$, we then get, using (ii) (a),

$$
U_{t} v:=\left(\varphi_{\varepsilon}\left(\frac{t}{n}\right)\right)^{n} v
$$

An application of (2.2) yields,

$$
\left\|U_{t} v\right\|=\|v\|
$$

Hence, $U_{t}$ extends by closure (since (i) holds and $\mathscr{D}$ is dense) to a unitary operator $U_{t}: \mathscr{H} \rightarrow \mathscr{H}$ for all $t \in \mathbb{R}$. By (2.2), the set of pairs in $\mathbb{R}$ for which the group

$$
U_{s+t}=U_{s} U_{t}
$$

holds is open, closed, and non-empty. Using (ii)(a) and connectedness of $\mathbb{R}$, we conclude that (2.5) holds for all $s, t \in \mathbb{R}$.

Step $3 t \longrightarrow U_{t}$ is strongly continuous: This follows from (ii)(b).

$\overline{\text { Step } 4}$ By Stone's theorem, $\left\{U_{t}\right\}_{t \in \mathbb{R}}$ has a unique infinitesimal generator, i.e., there is a selfadjoint operator $\widetilde{H}$ (generally unbounded) such that

$$
U_{t}=e^{i t \widetilde{H}}=\int_{\mathbb{R}} e^{i \lambda t} P(d \lambda)
$$

where $P(\cdot)$ denotes the spectral resolution of the selfadjoint operator $\widetilde{H}$.

Step 5 The operator $i \widetilde{H}$ extends $H$, i.e., $\mathscr{D}$ is contained in $\operatorname{dom}(\widetilde{H})$, and

$$
H v=i \widetilde{H} v=\lim _{t \rightarrow 0} \frac{U_{t} v-v}{t}
$$

holds for all $v \in \mathscr{D}$. 
Proof of (2.7). Pick $v \in \mathscr{D}, \varepsilon>0$ s.t. $v \in \mathscr{D}_{\varepsilon}$, see (i); then for $|t|<\varepsilon, \varphi_{\varepsilon}(t) v=U_{t} v$, and

$$
\left.\frac{d}{d t}\right|_{t=0} \varphi_{\varepsilon}(t) \underset{(\text { by }(\text { ii) }(\mathrm{b}))}{=} H v=\lim _{t \rightarrow 0} \frac{U_{t} v-v}{t}
$$

exists, and by definition of the infinitesimal generator of $\left\{U_{t}\right\}_{t \in \mathbb{R}}$, we get $H v=i \widetilde{H} v$ which is the desired conclusion (2.7).

We now show that the closure of $(H, \mathscr{D})$, the operator $H$ with $\mathscr{D}$ as its domain, is $i \widetilde{H}$, i.e., that infinitesimal generator of the unitary one-parameter group $\left\{U_{t}\right\}_{t \in \mathbb{R}}$ from (2.6)-(2.7) is the closure of $H$.

Details: Since we already proved that $i \widetilde{H}$ is an extension of $H$ on $\mathscr{D}$, i.e., that the closure of the graph

$$
G(H):=\left\{\left(\begin{array}{c}
v \\
H v
\end{array}\right), v \in \mathscr{D}\right\}
$$

in $\mathscr{H} \times \mathscr{H}$ is contained in the graph of $i \widetilde{H}$, and $G(H)^{c l}=G(i \widetilde{H})$; it is enough to prove that $(H, \mathscr{D})$ has deficiency indices $(0,0)$; for then the skew-adjoint extension $i \widetilde{H}$ is unique, so it must be the closure of $H$.

To verify the index assertion, we must show that the following two equations,

$$
\left\langle H v \pm v, f_{ \pm}\right\rangle \equiv 0, \forall v \in \mathscr{D}
$$

has only solution $f_{ \pm}=0$ in $\mathscr{H}$. By symmetry, we need only consider one of the equations in (2.8), say the $f_{-}$case:

For $v \in \mathscr{D}=\bigcup_{\varepsilon \in \mathbb{R}_{+}} \mathscr{D} \varepsilon$, we pick $\varepsilon$ and $\varepsilon^{\prime}$ as in (ii)(c) in the statement of the theorem. Using formula (2.3), we conclude that $U_{t} v \in \mathscr{D}$ for all $t \in \mathbb{R}$. Hence, by (2.8), we get

$$
\left\langle H U_{t} v, f_{-}\right\rangle=\left\langle U_{t} v, f_{-}\right\rangle, \forall t \in \mathbb{R} .
$$

But by (2.7) this is equivalent to the following differential equation:

$$
\frac{d}{d t}\left\langle U_{t} v, f_{-}\right\rangle=\left\langle U_{t} v, f_{-}\right\rangle, \forall t \in \mathbb{R},
$$

with initial condition $\left.\left\langle U_{t} v, f_{-}\right\rangle\right|_{t=0}=\left\langle v, f_{-}\right\rangle$. Hence

$$
\left\langle U_{t} v, f_{-}\right\rangle=e^{t}\left\langle v, f_{-}\right\rangle, \forall t \in \mathbb{R} .
$$

But since $U_{t}$ is unitary (isometric) the LHS in (2.9) is bounded as a function of $t \in \mathbb{R}$, while the RHS in (2.9) is always unbounded when $\left\langle v, f_{-}\right\rangle \neq 0$. We conclude therefore that $\left\langle v, f_{-}\right\rangle=0$ for all $v \in \mathscr{D}$. But, by condition (i) in the theorem, $\mathscr{D}$ is dense in $\mathscr{H}$, and so $f_{-}=0$.

Remark 2.2. The converse to the implication in Theorem 2.1 holds; in fact a slightly stronger version holds. I.e., it holds that every skew-adjoint operator $i \widetilde{H}\left(\widetilde{H}^{*}=\widetilde{H}\right)$ in a Hilbert space $\mathscr{H}$ admits dense subspaces $\mathscr{D}$ contained in $\operatorname{dom}(i \widetilde{H})$ which satisfy the conditions (i)-(ii) from the statement of Theorem 2.1.

In fact, there are many such choices for $\mathscr{D}$; and it becomes more of a question of identifying choices that are useful in applications. Below we sketch a choice of dense 
subspace $\mathscr{D}$ (subject to (i)-(ii)) for a given skew-adjoint operator $i \widetilde{H}$ in $\mathscr{H}$, where $\mathscr{H}$ is a fixed Hilbert space.

Given $i \widetilde{H}$, we get a projection valued measure $P(\cdot)$ as in $(2.6)$; i.e., $P(\cdot)$ is a sigmaadditive function defined on $\mathscr{B}(\mathbb{R})$, Borel sets, such that

$$
\begin{gathered}
P(\mathbb{R})=I_{\mathscr{H}} \\
P(A)=P(A)^{*}=P(A)^{2}, \forall A \in \mathscr{B}(\mathbb{R}) \\
P(A \cap B)=P(A) P(B), \forall A, B \in \mathscr{B}(\mathbb{R})
\end{gathered}
$$

and s.t. (2.6) holds.

We then set, for all $\varepsilon \in \mathbb{R}_{+}, \mathscr{D}_{\varepsilon}:=P\left(-\varepsilon^{-1}, \varepsilon^{-1}\right) \mathscr{H} \subset \mathscr{H}$, and $\mathscr{D}:=\bigcup_{\varepsilon \in \mathbb{R}_{+}} \mathscr{D}_{\varepsilon}$. It then follows from basic spectral theory that this $\mathscr{D}$ satisfies the conditions from (i)-(ii) in the statement of Theorem 2.1.

Example 2.3. Let $\mathscr{H}=L^{2}(0,1)$, and $\mathscr{D}=C_{c}^{1}(0,1)=$ compactly supported $C^{1}$ functions in $(0,1)$. And $H=d / d x$, skew-symmetric on $\mathscr{D}$. If $v$ is in $\mathscr{D}$ we can exponentiate locally as

$$
\varphi(t) v(\cdot):=v(\cdot-t)
$$

as long as we don't translate out of $(0,1)$, but $H$ is not essentially skew-adjoint; it has deficiency indices $(1,1)$. Note that $\varphi(t)$ does not satisfy the local invariance condition. Reason: The support of $v(\cdot-t)$ gets closer to a boundary point as $|t| \neq 0$.

To see that $H$ above with $\mathscr{D}=\operatorname{dom}(H)=C_{c}^{\infty}(0,1)$ has von Neumann indices $(1,1)$, note that each of the two equations

$$
H^{*} f_{ \pm}=\mp f_{ \pm}
$$

has non-zero solution, i.e., $f_{ \pm} \in L^{2}(0,1), f_{ \pm} \in \operatorname{dom}\left(H^{*}\right)$. The solutions are

$$
f_{ \pm}(x)=\text { const } \cdot e^{ \pm x}, x \in(0,1) \text {. }
$$

\section{COMMUTING SKEW-SYMMETRIC OPERATORS WITH COMMON DENSE DOMAIN}

Here we study the case of integrability and extendability of abelian Lie algebras of unbounded skew-symmetric operators with common dense domain in Hilbert space.

Theorem 3.1. Fix $n \in \mathbb{N}$, and set $J_{n}:=\{1, \ldots, n\}$. Let $H_{i}, i \in J$, be a set of skew-symmetric operators with a common dense domain $\mathscr{D}$ in a Hilbert space $\mathscr{H}$, i.e.,

$$
\left\langle H_{j} v, w\right\rangle+\left\langle v, H_{j} w\right\rangle=0
$$

for all $v, w \in \mathscr{D}, j \in J_{n}$. Suppose there are subspaces $\mathscr{D}_{\varepsilon}, \varepsilon \in \mathbb{R}_{+}$, such that

$$
\mathscr{D}=\bigcup_{\varepsilon \in \mathbb{R}_{+}} \mathscr{D}_{\varepsilon}
$$


(ii') For every $\varepsilon \in \mathbb{R}_{+}$there is an $\varepsilon^{\prime}, 0<\varepsilon^{\prime}<\varepsilon$, and there are operators

$$
\left\{\varphi_{\varepsilon, j}(t):|t|<\varepsilon\right\}
$$

with dense domain $\mathscr{D}$ such that:

For all $j \in J_{n}$,

(a) $\varphi_{\varepsilon, j}(s+t)=\varphi_{\varepsilon, j}(s) \varphi_{\varepsilon, j}(t),|s|<\varepsilon,|t|<\varepsilon,|s+t|<\varepsilon$;

(b) $\frac{d}{d t} \varphi_{\varepsilon, j}(t)=H_{j} \varphi_{\varepsilon, j}(t),|t|<\varepsilon^{\prime}, \varphi_{\varepsilon, j}(0)=I$;

(c) $\varphi_{\varepsilon, j}(t)$ leaves $\mathscr{D}_{\varepsilon}$ invariant for all $t \in\left(-\varepsilon^{\prime}, \varepsilon^{\prime}\right)$; and

(d)

$$
\varphi_{\varepsilon, j}(s) \varphi_{\varepsilon, j^{\prime}}(t)=\varphi_{\varepsilon, j^{\prime}}(t) \varphi_{\varepsilon, j}(s)
$$

for all $j, j^{\prime} \in J_{n},|s|,|t|<\varepsilon$.

Then the operators $H_{j}$ are essentially skew-adjoint, and the operator closures $\overline{H_{j}}$ strongly commute, i.e., the operators $\overline{H_{j}}$ have commuting spectral projections.

Proof. Note steps 1-5 in the proof of Theorem 2.1 carry over for each $H_{j}, j \in J_{n}$.

Indeed, for each $j \in J_{n}$, define $U_{j}(t)$ as in (2.3), acting on $\mathscr{D}$. Then $U_{j}(t)$ is norm-preserving (see (2.4)), and by (ii')(d), we have

$$
U_{j}(t) U_{j^{\prime}}(s)=U_{j^{\prime}}(s) U_{j}(t) \text { on } \mathscr{D}, \forall j, j^{\prime} \in J_{n}, t, s \in \mathbb{R} \text {. }
$$

Therefore, the operators $U_{j}$ extend by continuity to a family of commuting unitary one-parameter groups in $\mathscr{H}$. By Stone's theorem,

$$
U_{j}(t)=e^{i t \widetilde{H_{j}}}=\int e^{i t \lambda} P_{j}(d \lambda), t \in \mathbb{R}
$$

where $\widetilde{H}_{j}$ is the corresponding selfadjoint infinitesimal generator, and $P_{j}$ the projection valued measure. Moreover, $H_{j} \subset i \widetilde{H}_{j}$ and $\overline{H_{j}}=i \widetilde{H}_{j}$, i.e., $H_{j}$ is essentially selfadjoint.

For finish the proof, we recall a general theorem in the theory of integrable representations of $*$-algebras. See [Jør76, Lemma 1] and a complete proof in [JM84].

Details: Note (i')-(ii') define a representation $\rho$ of an $n$-dimensional abelian Lie algebra $\mathfrak{g}\left(=\mathbb{R}^{n}\right)$ acting on the common dense domain $\mathscr{D}$ in $\mathscr{H}$. By [Jør76, Lemma 1], the local invariance condition and the density of $\mathscr{D}$ imply that $\rho$ can be exponentiated (i.e., $\rho$ is integrable) to a unitary representation $\mathcal{U}$ of the Lie group $G=\left(\mathbb{R}^{n},+\right)$, and $\rho=d \mathcal{U}$. Therefore, the generators $i \widetilde{H}_{j}$ strongly commute.

Combining the ideas above, we get the following result for commuting operators. We state it for $n=2$, but the conclusions hold mutatis mutandis for the case $n>2$ as well.

Proposition 3.2. Let $H_{1}$ and $H_{2}$ be two skew-symmetric operators defined on a common dense domain $\mathscr{D}$ in a Hilbert space $\mathscr{H}$. Assume that $H_{j} \mathscr{D} \subseteq \mathscr{D}, j=1,2$. Then the following conditions are equivalent:

(1) the operator $L:=H_{1}^{2}+H_{2}^{2}$ is essentially selfadjoint on $\mathscr{D}$;

(2) each operator $H_{j}$ is essentially skew-adjoint and the two unitary one-parameter groups $U_{j}(t):=e^{t \overline{H_{j}}}, j=1,2$ are commuting; 
(3) each operator $H_{j}$ is essentially skew-adjoint and

$$
U\left(t_{1}, t_{2}\right):=e^{t_{1} \overline{H_{1}}} e^{t_{2} \overline{H_{2}}},\left(t_{1}, t_{2}\right) \in \mathbb{R}^{2}
$$

defines a strongly continuous unitary representation of $\left(\mathbb{R}^{2},+\right)$ acting on $\mathscr{H}$;

(4) for each $j$ and $\lambda \in \mathbb{C} \backslash i \mathbb{R}$, the operator ranges $\left(\lambda-H_{j}\right) \mathscr{D}$ are dense in $\mathscr{H}$, and the bounded operators

$$
\left(\lambda_{1}-H_{1}\right)^{-1} \text { and }\left(\lambda_{2}-H_{2}\right)^{-1}
$$

are commuting, $\forall \lambda_{j} \in \mathbb{C} \backslash i \mathbb{R}$; and

(5) the conditions ( $\left.i^{\prime}\right)$ and (ii') in Theorem 3.1 hold.

\section{Lie Algebras, AND local RePresentations of Lie groups}

We begin with rigorous definitions of the following two notions: integrability and extendability for Lie algebras $\mathfrak{g}$ of unbounded skew-symmetric operators with common dense domain in Hilbert space. In our main result, Corollary 4.16, we show that a given finite-dimensional Lie algebra $\mathfrak{g}$ of skew-symmetric operators is integrable to a unitary representation of the corresponding simply connected Lie group if and only if it has a dense and locally invariant domain.

Definition 4.1. Let $\mathscr{H}$ be a Hilbert space, and $\mathscr{D}$ a dense subspace. Let $\mathfrak{g}$ be a finite dimensional Lie algebra over $\mathbb{R}$. Let $S k(\mathscr{D})$ denote the real Lie algebra of all linear operators $X$ satisfying

(i) $\mathscr{D} \subset \operatorname{dom}(X)$, for all $X \in \mathfrak{g}$;

(ii) $X(\mathscr{D}) \subset \mathscr{D}$;

(iii) $\langle X u, w\rangle+\langle u, X w\rangle=0$, for all $X \in \mathfrak{g}$, and $u, w \in \mathscr{D}$.

A representation $\rho$ of $\mathfrak{g}$ is a Lie-homomorphism $\rho: \mathfrak{g} \rightarrow S k(\mathscr{D})$, i.e.,

$$
\rho([x, y])=[\rho(x), \rho(y)], \forall x, y \in \mathfrak{g} .
$$

Occasionally, we shall use the notation $X=\rho(x), x \in \mathfrak{g}$.

Definition 4.2. We say that a representation $(\rho, \mathfrak{g}, \mathscr{D})$ has an integrable extension iff (Def) there is a unitary representation $\mathcal{U}$ of the simply connected Lie group $G$ with $\mathfrak{g}$ as its Lie algebra, s.t.

$$
\rho(x) \subseteq d \mathcal{U}(x), \forall x \in \mathfrak{g},
$$

where the containment " $\subseteq$ " in (4.2) refers to containment of graphs, i.e.,

$$
\begin{array}{r}
\mathscr{D} \subseteq \operatorname{dom}(\mathcal{U}(x)), \forall x \in \mathfrak{g}, \text { and } \\
\rho(x) w=d \mathcal{U}(x) w, \forall w \in \mathscr{D} .
\end{array}
$$

Definition 4.3. We say that a Lie algebra representation $(\rho, \mathfrak{g}, \mathscr{D})$ is integrable if $(4.2)$ holds, but with equality for the closure, i.e.,

$$
\begin{aligned}
\operatorname{Graph}(\rho(x))^{\mathscr{H} \times \mathscr{H} \text { closure }} & =\operatorname{Graph}(d \mathcal{U}(x)), \text { where } \\
d \mathcal{U}(x) w & =\lim _{t \rightarrow 0} \frac{\mathcal{U}(\exp t x) w-w}{t}
\end{aligned}
$$


Lemma 4.4 ([JM84]). Let $G$ be a Lie group with Lie algebra $\mathfrak{g}$, and exponential mapping $\mathfrak{g} \stackrel{\exp }{\longrightarrow} G$, and let $\mathcal{U}$ be a unitary representation of $G$ acting on a Hilbert space $\mathscr{H}$. Set

$$
\mathscr{H}_{\infty}:=\left\{w \in \mathscr{H} \mid(G \ni g \rightarrow \mathcal{U}(g) w) \in C^{\infty}(G, \mathscr{H})\right\},
$$

the $C^{\infty}$-vectors of $\mathcal{U}$; then

$$
d \mathcal{U}(x) w=\lim _{t \rightarrow 0} \frac{\mathcal{U}(\exp t x) w-w}{t}
$$

is well defined for all $w \in \mathscr{H}_{\infty}, x \in \mathfrak{g}$.

Moreover, $\mathrm{d} \mathcal{U}(x)$ on $\mathscr{H}_{\infty}$ is essentially skew-adjoint; i.e.,

$$
\left(\left.d \mathcal{U}(x)\right|_{\mathscr{H}}\right)^{*}=-d \mathcal{U}(x), \forall x \in \mathfrak{g} .
$$

Note. If $X$ is an operator with dense domain, then domain of its adjoint $X^{*}$ is $\left\{\overline{w \in \mathscr{H}} \mid \exists C=C_{w}<\infty\right.$ s.t. $\left.|\langle w, X u\rangle| \leq C\|u\|, \forall u \in \operatorname{dom}(X)\right\}$.

Example 4.5. Let $H_{1}=\left.\frac{d}{d x}\right|_{C_{c}^{\infty}(0,1)}$ in $L^{2}(0,1)$. $H$ is densely defined, skew-symmetric, with deficiency indices $(1,1) . \stackrel{H_{1}}{H}$ is extendable but not integrable.

On the other hand, $H_{2}=\left.\frac{d}{d x}\right|_{C_{c}^{\infty}(\mathbb{R})}$ is densely defined, skew-symmetric, acting in $L^{2}(\mathbb{R})$, and it has deficiency indices $(0,0)$; i.e., ${\overline{H_{2}}}^{*}=-\overline{H_{2}}$, skew-adjoint. $\overline{H_{2}}$ generates the one-parameter unitary group $\left\{U(t)=e^{-t \overline{H_{2}}}\right\}_{t \in \mathbb{R}}$, where

$$
U(t) f(x)=f(x-t)
$$

for all $f \in L^{2}(\mathbb{R})$. Therefore, $H_{2}$ is integrable.

Example 4.6. Let $M$ denote the Riemann surface of the complex $\log z$ function. We will realize $M$ as a covering space for $\mathbb{R}^{2} \backslash\{(0,0)\}$ with an infinite number of sheets indexed by $\mathbb{Z}$ as follows (see Fig 4.1):
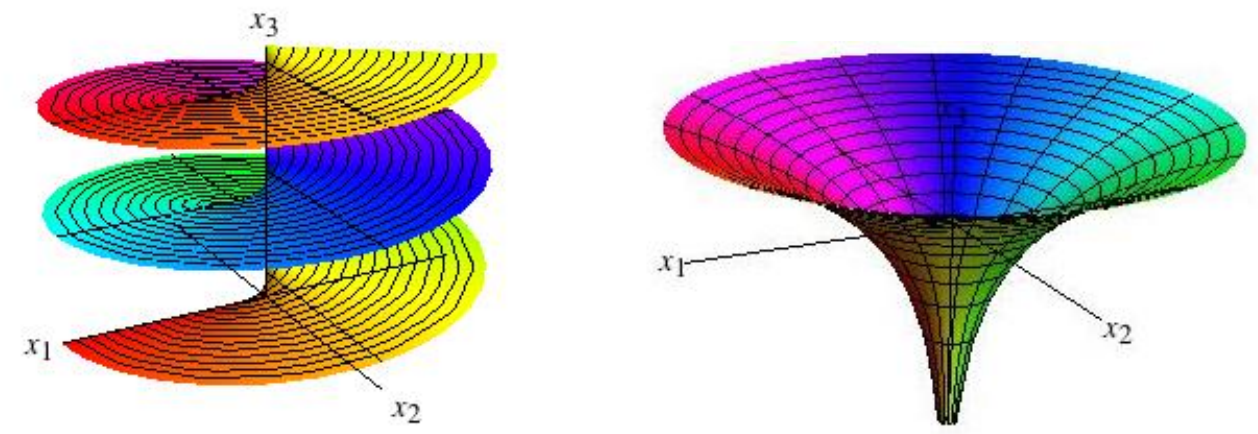

Figure 4.1. $M$ the Riemann surface of $\log z$ as an $\infty$ cover of $\mathbb{R}^{2} \backslash\{(0,0)\}$.

Rotate the real $\log x, x \in \mathbb{R}_{+}$, in the $\left(x_{1}, x_{2}\right)$ plane while creating spirals in the $x_{3}$-direction, one full rotation for each interval $n \leq x_{3}<n+1, n \in \mathbb{Z}$. 
The measure of $L^{2}(M)$ and $C_{c}^{\infty}(M)$ derive from the i.e., the 2D-Lebesgue measure lifts to a unique measure on $M$; hence $L^{2}(M)$. Here the two skew symmetric operators $\frac{\partial}{\partial x_{j}}, j=1,2$ with domain $C_{c}^{\infty}(M)$ define an abelian 2-dimensional Lie algebra of densely defined operators in the Hilbert space $L^{2}(M)$.

Proposition 4.7. (i) The $\left\{\frac{\partial}{\partial x_{j}}\right\}_{j=1,2}$ Lie algebra with domain $C_{c}^{\infty}(M) \subset L^{2}(M)$ is not extendable (see Definition 4.2).

(ii) Each operator $\frac{\partial}{\partial x_{j}}$ on $C_{c}^{\infty}(M)$ is essentially skew-adjoint, i.e.,

$$
-\left(\left.\frac{\partial}{\partial x_{j}}\right|_{C_{c}^{\infty}(M)}\right)^{*}=\operatorname{closure}\left(\left.\frac{\partial}{\partial x_{j}}\right|_{C_{c}^{\infty}(M)}\right), j=1,2 .
$$

(iii) The two skew-adjoint operators in (4.7) are not strongly commuting.

(iv) The operator

$$
L:=\left(\frac{\partial}{\partial x_{1}}\right)^{2}+\left(\frac{\partial}{\partial x_{2}}\right)^{2} \text { on } C_{c}^{\infty}(M)
$$

has deficiency indices $(\infty, \infty)$.

Proof. The two operators $\frac{\partial}{\partial x_{j}}$ generate unitary one-parameter groups $U_{j}(t), j=1,2$, acting on $L^{2}(M)$ since the two coordinate translations

$$
\begin{aligned}
& \left(x_{1}, x_{2}\right) \longmapsto\left(x_{1}+t, x_{2}\right), x_{2} \neq 0 \\
& \left(x_{1}, x_{2}\right) \longmapsto\left(x_{1}, x_{2}+t\right), x_{1} \neq 0
\end{aligned}
$$

lift to unitary one-parameter groups acting on $L^{2}(M)$; and it is immediate that the respective infinitesimal generators are the closed operators $\frac{\partial}{\partial x_{j}}$.

If $\varphi \in C_{c}^{\infty}(M)$ is supported over some open set in $\mathbb{R}^{2} \backslash\{(0,0)\}$, for example, $\left(x_{1}-2\right)^{2}+x_{2}^{2}<1$, if $1<s<2,1<t<2$, then the two functions

$$
U_{1}(s) U_{2}(t) \varphi \text { and } U_{2}(t) U_{1}(s) \varphi
$$

are supported on different sheets in the covering $M \longrightarrow \mathbb{R}^{2} \backslash\{(0,0)\}$, two levels opposite; see Fig 4.2. Hence the two unitary groups $\left\{U_{1}(s)\right\}_{s \in \mathbb{R}}$ and $\left\{U_{2}(t)\right\}_{t \in \mathbb{R}}$ do not commute.

Hence it follows from Nelson's theorem [Nel59] that $L$ in (4.8) is not essentially selfadjoint. Since $L \leq 0$ (in the sense of Hermitian operators) its deficiency indices are equal. It was proved in [Tia11] that the indices are $(\infty, \infty)$; see also details below. 


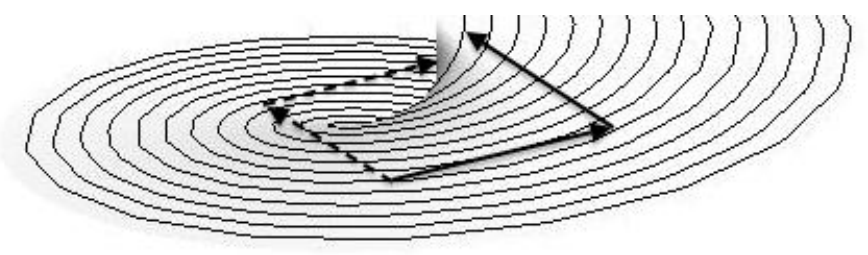

Figure 4.2. Translation of $\varphi$ to different sheets.

Proposition 4.8. Let $M$ be the $\infty$-covering Riemann surface of $\log z$, and let

$$
\left\{U_{1}(s)\right\}_{s \in \mathbb{R}},\left\{U_{2}(t)\right\}_{t \in \mathbb{R}}
$$

be the two unitary one-parameter groups in $L^{2}(M)$ from Proposition 4.7. Then the two groups together act irreducibly on $L^{2}(M)$.

Proof. We shall need the following lemma.

Lemma 4.9. Let $\varepsilon, s, t \in \mathbb{R}_{+}$, and pick a sheet $M_{k}$ of the surface $M$ (see Fig 4.1). On $M_{k}$, consider the "box" $B_{s, t}=\left\{\left(x_{1}, x_{2}\right) \mid \varepsilon<x_{1}<s, \varepsilon<x_{2}<t\right\}$, then the commutator

$$
C(s, t)=U_{1}(s) U_{2}(t) U_{1}(-s) U_{2}(-t)
$$

acts as the identity operator in $B_{s, t}$.

Proof. This follows from the reasoning below (4.11) and Fig 4.2.

Proof of Proposition 4.8 continued. If $P$ is a projection in $L^{2}(M)$ which commutes with all the operators $C(s, t)$ in (4.12), then $P$ must be a multiplication operator; say multiplication by some function $f, f=\bar{f}=f^{2}$. If $P$ also commutes with each of the unitary one-parameter groups $\left\{U_{1}(s)\right\}$ and $\left\{U_{2}(t)\right\}$, it follows from that (4.9)-(4.10) that the function $f$ must be constant; hence $f \equiv 0$, or $f \equiv 1$. Consequently $P=0$, or $P=I_{L^{2}(M)}$, and it follows that the system $\left\{U_{1}(s), U_{2}(t)\right\}$ is irreducible.

Let $X_{j}=\left.\frac{\partial}{\partial x_{j}}\right|_{C^{\infty}(M)}, j=1,2$, as in Proposition 4.7. We see that each $X_{j}$ is essentially skew-adjoint, but $e^{t \overline{X_{1}}} e^{s \overline{X_{2}}} \neq e^{s \overline{X_{2}}} e^{t \overline{X_{1}}}$ globally. Set

$$
\mathscr{D}_{\infty}:=\bigcap_{l_{1}, l_{2} \in \mathbb{N}} \mathscr{D}\left(\bar{X}_{1}^{l_{1}} \bar{X}_{2}^{l_{2}}\right)=\bigcap_{n=1}^{\infty} \operatorname{dom}\left(\bar{L}^{n}\right)
$$

where $l_{1}, l_{2} \in \mathbb{N}$. Let

$$
\begin{aligned}
L & :=\left.\Delta\right|_{\mathscr{D}_{\infty}} \\
L^{\prime} & :=\left.\Delta\right|_{C_{c}^{\infty}(M)}
\end{aligned}
$$

where $\Delta=\left(\frac{\partial}{\partial x_{1}}\right)^{2}+\left(\frac{\partial}{\partial x_{2}}\right)^{2}$. 
By Nelson's theorem [Nel59], we have the following equivalence

$$
\text { the two operators } \begin{gathered}
\overline{X_{1}}, \overline{X_{2}} \text { commute strongly } \\
\Uparrow \\
L^{*}=L
\end{gathered}
$$

Since $L \leq 0$, it suffices to consider the deficiency space

$$
\mathscr{D}_{1}(L):=\left\{\psi \in \mathscr{D}\left(L^{*}\right): L^{*} \psi=\psi\right\} .
$$

By elliptic regularity, we have $\psi \in C^{\infty}(M) \cap L^{2}(M)$, for all $\psi \in \mathscr{D}_{1}(L)$.

Remark 4.10. There is a distinction between the two versions of Laplacian. For example, if $M=\mathbb{R}^{2} \backslash\{(0,0)\}$, i.e., the punctured plane, then $\mathscr{D}_{\infty}=C^{\infty}$-vectors for the unitary representation of $G=\left(\mathbb{R}^{2},+\right)$ on $L^{2}(M)$. In this case, $L$ is the free Hamiltonian which has deficiency indices $(0,0)$, however $L^{\prime}$ has indices $(1,1)$. The corresponding unitary groups are translations in the two coordinate directions of $\mathbb{R}^{2}$, and they do commute. Therefore, $\overline{X_{1}}$ and $\overline{X_{2}}$ strongly commute, and the two dimension Lie algebra with generators $X_{j}$ is integrable.

Since in the $\log z$ example, $\overline{X_{1}}$ and $\overline{X_{2}}$ do not strongly commute, it follows that $X_{1}^{2}+X_{2}^{2}$ has deficiency indices $(m, m), m>0$. We show below that $m=\infty$ [Tia11].

Lemma 4.11. Let $K_{\nu}$ be the modified Bessel function of the second kind of order $\nu$, and suppose $\nu \in(-1,1)$. Then

$$
\int_{0}^{\infty}\left|K_{\nu}(z)\right|^{2} z d z=\frac{1}{2} \frac{\pi \nu}{\sin \pi \nu} .
$$

Proof. By Nicholson's integral representation of $K_{\nu}$ [Wat44, pg. 440], we have

$$
K_{\mu}(z) K_{\nu}(z)=2 \int_{0}^{\infty} K_{\mu+\nu}(2 z \cosh t) \cosh ((\mu-\nu) t) d t .
$$

Since $K_{\nu}$ is real-valued, setting $\mu=\nu$, it follows that

$$
\left|K_{\nu}(z)\right|^{2}=2 \int_{0}^{\infty} K_{2 \nu}(2 z \cosh t) d t
$$

We recall the following identity [Wat44, pg. 388, eq. (8)]:

$$
\int_{0}^{\infty} K_{\nu}(z) z^{\beta-1} d z=2^{\beta-2} \Gamma\left(\frac{\beta+\nu}{2}\right) \Gamma\left(\frac{\beta-\nu}{2}\right), \Re(\beta)>|\Re(\nu)| .
$$

For $\nu \in(-1,1), \beta=2$, (4.20) yields

$$
\begin{aligned}
\int_{0}^{\infty} K_{2 \nu}(2 z \cosh t) z d z & =\frac{1}{(2 \cosh t)^{2}} \Gamma\left(\frac{2+2 \nu}{2}\right) \Gamma\left(\frac{2-2 \nu}{2}\right) \\
& =\frac{1}{(2 \cosh t)^{2}} \Gamma(1+\nu) \Gamma(1-\nu) \\
& =\frac{1}{(2 \cosh t)^{2}} \nu \Gamma(\nu) \Gamma(1-\nu)
\end{aligned}
$$




$$
=\frac{1}{(2 \cosh t)^{2}}\left(\frac{\pi \nu}{\sin \pi \nu}\right)
$$

where the last step follows from the identity

$$
\Gamma(\nu) \Gamma(1-\nu)=\frac{\pi}{\sin \pi \nu}
$$

of the Gamma function $\Gamma$.

Combining (4.19) and (4.21), and using Fubinis's theorem, we get

$$
\begin{aligned}
\int_{0}^{\infty}\left|K_{\nu}(z)\right|^{2} z d z & =2 \int_{0}^{\infty}\left(\int_{0}^{\infty} K_{2 \nu}(2 z \cosh t) z d z\right) d t \\
& =2\left(\frac{\pi \nu}{\sin \pi \nu}\right) \int_{0}^{\infty} \frac{1}{(2 \cosh t)^{2}} d t \\
& =\frac{1}{2} \frac{\pi \nu}{\sin \pi \nu} \int_{0}^{\infty} \frac{1}{\cosh ^{2} t} d t \\
& =\frac{1}{2} \frac{\pi \nu}{\sin \pi \nu}
\end{aligned}
$$

Let $M=$ the Riemann surface of complex $\log z$. Note that $M$ is covered by a single coordinate patch under polar coordinates, i.e.,

$$
\begin{aligned}
& x=r \cos \theta \\
& y=r \sin \theta
\end{aligned}
$$

where $r \in \mathbb{R}_{+}, \theta \in \mathbb{R}$; and it has the standard metric

$$
d s^{2}=d r^{2}+r^{2} d \theta^{2}
$$

Taking Fourier transform in the $\theta$ variable leads to the decomposition

$$
\begin{aligned}
L^{2}(M) & =\int_{\mathbb{R}}^{\oplus} \mathscr{H}_{\xi} d \xi, \text { where } \\
\mathscr{H}_{\xi} & :=L^{2}\left(\mathbb{R}_{+}, r d r\right) \otimes \operatorname{span}\left\{e^{i \xi \theta}\right\} .
\end{aligned}
$$

Specifically, for all $f \in L^{2}(M)$, we set

$$
\begin{aligned}
\widehat{f}(r, \xi) & :=\int_{-\infty}^{\infty} f(r, \theta) e^{-i \xi \theta} d \theta ; \text { then } \\
f(r, \theta) & =\frac{1}{2 \pi} \int_{-\infty}^{\infty} \widehat{f}(r, \xi) e^{i \theta \xi} d \xi, \text { and } \\
\|f\|_{L^{2}(M)}^{2} & =\frac{1}{2 \pi} \int_{-\infty}^{\infty}\left(\int_{0}^{\infty}|\widehat{f}(r, \xi)|^{2} r d r\right) d \xi
\end{aligned}
$$

The formal 2 dimensional Laplacian takes the form

$$
\Delta=\int^{\oplus}\left(\frac{1}{r} \frac{d}{d r}\left(r \frac{d}{d r}\right)-\frac{\xi^{2}}{r^{2}}\right) \otimes 1 .
$$


Proposition 4.12. Let $L$ be the Laplacian in (4.14), and $\mathscr{D}_{1}(L)$ the deficiency space (4.16) as before. Then, $\psi \in \mathscr{D}_{1}(L)$, i.e., $\psi$ is a solution to the following equation

$$
\Delta \psi=\psi, \psi \in C^{\infty}(M) \cap L^{2}(M)
$$

iff there is a Borel function $g$ supported in $(-1,1)$ and satisfies

$$
\int_{-1}^{1} \frac{1}{2} \frac{\pi \xi}{\sin \pi \xi}|g(\xi)|^{2} d \xi<\infty
$$

such that

$$
\psi(r, \theta)=\frac{1}{2 \pi} \int_{-1}^{1} g(\xi) K_{\xi}(r) e^{i \xi \theta} d \xi
$$

Here, $K_{\xi}$ is the modified Bessel function of the second kind. Consequently, L has deficiency indices $(\infty, \infty)$.

Proof. Let $\psi \in C^{\infty}(M) \cap L^{2}(M)$. Using the decomposition (4.23)-(4.24), we have

$$
\psi(r, \theta)=\frac{1}{2 \pi} \int_{-\infty}^{\infty}\left(\int_{0}^{\infty} \widehat{\psi}(r, \xi) e^{i \xi \theta} r d r\right) d \xi .
$$

It follows from (4.25) that

$$
\begin{aligned}
\Delta \psi & =\frac{1}{2 \pi} \int_{-\infty}^{\infty}\left(\int_{0}^{\infty} \Delta\left(\widehat{\psi}(r, \xi) e^{i \xi \theta}\right) r d r\right) d \xi \\
& =\frac{1}{2 \pi} \int_{-\infty}^{\infty}\left(\int_{0}^{\infty}\left(\frac{1}{r} \frac{d}{d r}\left(r \frac{d}{d r}\right)-\frac{\xi^{2}}{r^{2}}\right) \widehat{\psi}(r, \xi) e^{i \xi \theta} r d r\right) d \xi .
\end{aligned}
$$

Hence $\Delta \psi=\psi$ iff

$$
\left(\frac{1}{r} \frac{d}{d r}\left(r \frac{d}{d r}\right)-\frac{\xi^{2}}{r^{2}}\right) \widehat{\psi}(r, \xi)=\widehat{\psi}(r, \xi)
$$

where

$$
l_{\xi}:=\frac{1}{r} \frac{d}{d r}\left(r \frac{d}{d r}\right)-\frac{\xi^{2}}{r^{2}}
$$

is the Bessel differential operator of order $\xi$, acting in $L^{2}\left(\mathbb{R}_{+}, r d r\right)$.

Note that $\left.l_{\xi}\right|_{C_{c}^{\infty}\left(\mathbb{R}_{+}\right)} \leq 0$ (in the order of Hermitian operators); and it is essentially selfadjoint in $L^{2}\left(\mathbb{R}_{+}, r d r\right)$ iff $|\xi| \geq 1$, see e.g., [AG93]. Thus in the solution to (4.28), we must have $\xi \in(-1,1)$. The corresponding defect vector is given by

$$
\widehat{\psi}(r, \xi)=\text { const } \cdot K_{\xi}(r) ;
$$

where $K_{\xi}$ denotes the modified Bessel function of the second kind. Since the constant depends on $\xi$, we may write

$$
\widehat{\psi}(r, \xi)=g(\xi) K_{\xi}(r)
$$

subject to the condition

$$
\int_{-1}^{1}\left(\int_{0}^{\infty}\left|g(\xi) K_{\xi}(r)\right|^{2} r d r\right) d \xi<\infty
$$


By Fubini's theorem,

$$
\begin{aligned}
\operatorname{LHS}_{(4.29)} & =\int_{-1}^{1}\left(\int_{0}^{\infty}\left|K_{\xi}(r)\right|^{2} r d r\right)|g(\xi)|^{2} d \xi \\
& =\int_{-1}^{1} \frac{1}{2} \frac{\pi \xi}{\sin \pi \xi}|g(\xi)|^{2} d \xi, \text { by Lemma 4.11; }
\end{aligned}
$$

which gives (4.26).

Finally, we have

$$
\begin{aligned}
\psi(r, \theta) & =\frac{1}{2 \pi} \int_{-\infty}^{\infty} \widehat{\psi}(r, \xi) e^{i \xi \theta} d \xi \\
& =\frac{1}{2 \pi} \int_{-1}^{1} g(\xi) K_{\xi}(r) e^{i \xi \theta} d \xi
\end{aligned}
$$

which is the desired result.

Remark 4.13. The harmonic analysis of the Riemann surface $M$ of $\log z$ is of independent interest, but it will involve von Neumann algebras and non-commutative geometry. As we noted, to study this, we are faced with two non-commuting unitary one-parameter groups acting on $L^{2}(M)$ (corresponding to the two coordinates for $M$ ). Of interest here is the von Neumann algebra generated by these two non-commuting unitary one parameter groups. It is likely that this von Neumann algebra is a type III factor. There is a sequence of interesting papers by K. Schmudgen on dealing with some of this [Sch84, SF84, Sch85, Sch86b, Sch86a].

In any case, the properties of the von Neumann algebra depend on the defect space (4.16) for the $M$-Laplacian $L$. The role of the non-commutativity is tied in with the operator $L$ as follows:

Nelson's theorem on analytic vectors [Nel59] applies more generally to Lie algebras of operators, commutative or not. We summarize briefly its relevance. Let $\mathfrak{g}$ be a finitedimensional real Lie algebra of skew symmetric operators with a common dense domain in a fixed Hilbert space. Pick a basis for $\mathfrak{g}$, and let $L$ be the sum of squares of the basiselements; the Nelson-Laplacian. The first theorem in [Nel59] states that $L$ analytically dominates the Lie algebra $\mathfrak{g}$. The notion of "analytic domination" is powerful. It means that analytic vectors for $L$ are also analytic for the whole Lie algebra $\mathfrak{g}$. As a Corollary: If $L$ is essentially selfadjoint, it has a dense space of analytic vectors, and so these will also be analytic for $\mathfrak{g}$, and so $\mathfrak{g}$ is integrable.

It the commutative case, if $\operatorname{dim} \mathfrak{g}=n$, and if $L$ is essentially selfadjoint, then it follows that the Lie algebra $\mathfrak{g}$ is integrable; i.e., we have a unitary representation $\mathcal{U}$ of $\mathbb{R}^{n}$ such that $d \mathcal{U}=\mathfrak{g}$; see Definition 4.3. Hence the operators in $\mathfrak{g}$ are essentially skew-adjoint, and they strongly commute. The converse implication holds as well.

Returning to $L^{2}(M)$ : Since in our $\log z$ example (Examples 4.5-4.6), the two unitary one-parameter groups do not commute, it follows that $\mathfrak{g}$ is not integrable, and so the Nelson-Laplacian $L$ must have indices $(m, m), m>0$. 
Theorem 4.14 ([Jør76]). Let $\mathscr{H}$ be a Hilbert space. Let $\mathfrak{g} \subset \mathscr{L}(\mathscr{H})$ be a finite dimensional Lie algebra. Suppose $\mathfrak{g}$ is generated by a subset $S$ such that every $A \in S$ is closable and the closure $\bar{A}$ generates a $C_{0}$ group $\{\pi(t, A)\}_{t \in \mathbb{R}} \subset \mathscr{L}(\mathscr{H})$. Then $\mathfrak{g}$ is integrable iff $\mathfrak{g}$ has a dense locally invariant $\mathscr{D}$ in $\mathscr{H}$.

Remark 4.15. Theorem 4.14 also applies to abelian Lie algebras. In Example 4.6, we have two derivative operators acting on $L^{2}(M)$, where $M$ is the Riemann surface of $\log z$. The Lie algebra is two dimensional, and not integrable. We conclude that there is no locally invariant $\mathscr{D}$ for the two operators.

There is a local representation in $L^{2}(M)$ but not a global one. By global we mean the closures are strongly commuting, which we do not have. In our $\log z$ example it is obvious that we can integrate locally $\varphi_{g}, g$ in a small neighborhood of 0 in $\mathbb{R}^{2}$, so a local representation $\varphi$ of $\mathbb{R}^{2}$ acting on $L^{2}(M)$, but $\varphi$ will not have any locally invariant $\mathscr{D}$. This is different from our 1D examples. In Example 4.5, we had a $(1,1)$ example, but it is contained in a $(0,0)$ example which has a locally invariant $\mathscr{D}$. No such thing happens for $L^{2}(M)$ since the two operators are already essentially skew-adjoint.

Corollary 4.16. Let $G$ be a simply connected Lie group with Lie algebra $\mathfrak{g}$, and exponential mapping $\mathfrak{g} \stackrel{\exp }{\longrightarrow} G$. Let $\mathscr{H}$ be a Hilbert space, and $\mathscr{D} \subset \mathscr{H}$ a dense subspace. Let $\rho \in S k(\mathscr{D}, \mathscr{H})$ be a representation of $\mathfrak{g}$ with $\mathscr{D}$ as a common dense domain for the skew-symmetric operators $\{\rho(x) \mid x \in \mathfrak{g}\}$. By a local representation for $\rho$, we mean a neighborhood $W$ of e in $G$ and a mapping

$$
\varphi_{W}: W \longrightarrow \text { operators on } \mathscr{D} \text { (generally unbounded) }
$$

such that if $g_{1}, g_{2}$ and $g_{1} g_{2}$ are in $W$, then the following two conditions

$$
\begin{gathered}
\varphi_{W}\left(g_{1} g_{2}\right)=\varphi_{W}\left(g_{1}\right) \varphi_{W}\left(g_{2}\right), \text { and } \\
\frac{d}{d t} \varphi_{W}(\exp (t x))=\rho(x) \varphi_{W}(\exp (t x))
\end{gathered}
$$

hold where $x \in \mathfrak{g}$, and $\exp (t x) \in W$.

Suppose that there is a system of neighborhoods $\mathcal{W}=\{W\}$ of e in $G$, solutions $\varphi_{W}$ to (4.30)-(4.32), and subspaces $\mathscr{D}_{W} \subset \mathscr{D}$ such that the following two conditions hold:

$$
\begin{gathered}
\mathscr{D}=\bigcup_{W \in \mathcal{W}} \mathscr{D}_{W}, \text { and } \\
\varphi_{W}(g) \mathscr{D}_{W} \subset \mathscr{D}_{W}, \forall g \in W .
\end{gathered}
$$

Then we conclude that $\rho$ is integrable, i.e., there is a unitary representation $\mathcal{U}$ of $G$, acting on $\mathscr{H}$, such that

$$
\mathscr{D} \subseteq \mathscr{H}_{\infty}, \rho(x) \subseteq d \mathcal{U}(x), \forall x \in \mathfrak{g} ; \text { and }
$$

$($ Graph closure of $\rho(x))=d \mathcal{U}(x), \forall x \in \mathfrak{g}$. 


\section{Appendix. RiemanN SURfaCes of Finite-COVER DEgREe}

Fix $N \in \mathbb{N}$, and let $M$ be the $N$-covering surface of $\mathbb{R}^{2} \backslash\{(0,0)\}$. Under polar coordinates, $M$ is covered in a single coordinate patch as

$$
\begin{aligned}
& x=r \cos \theta \\
& y=r \sin \theta
\end{aligned}
$$

where $r \in \mathbb{R}_{+}$, and $\theta \in[0,2 \pi N)$; and it has the induced metric

$$
d s^{2}=d r^{2}+r^{2} d \theta^{2}
$$

with volume form

$$
d V=r d r d \theta
$$

Using Fourier series in the $\theta$ variable, we have the following decomposition

$$
L^{2}(M)=\sum_{k \in \mathbb{Z}}^{\oplus}\left(L^{2}\left(\mathbb{R}_{+}, r d r\right) \otimes \operatorname{span}\left\{e^{i \theta k / N}\right\}\right) .
$$

See, for example, [SW71, Chap. 4]. Hence, for all $f \in L^{2}(M)$, we set

$$
\begin{aligned}
\widehat{f}_{k}(r) & =\frac{1}{2 \pi N} \int_{0}^{2 \pi N} f(r, \theta) e^{-i \theta k / N} d \theta ; \text { then } \\
f(r, \theta) & =\sum \widehat{f}_{k}(r) e^{i \theta k / N}
\end{aligned}
$$

and

$$
\|f\|_{L^{2}(M)}^{2}=\sum_{k=-\infty}^{\infty} \int_{0}^{\infty}\left|\widehat{f}_{k}(r)\right|^{2} r d r .
$$

The formal 2D Laplacian in polar coordinates takes the form

$$
\Delta=\sum_{k \in \mathbb{Z}}^{\oplus}\left(\frac{1}{r} \frac{d}{d r}\left(r \frac{d}{d r}\right)-\frac{(k / N)^{2}}{r^{2}}\right) \otimes 1
$$

Set $W: L^{2}\left(\mathbb{R}_{+}, r d r\right) \rightarrow L^{2}\left(\mathbb{R}_{+}, d r\right)$ by

$$
W f(r):=r^{1 / 2} f(r) .
$$

$W$ is unitary and it converts (4.36) into

$$
\begin{aligned}
W \Delta W^{*} & =\sum_{k \in \mathbb{Z}}^{\oplus}\left(l_{k / N} \otimes 1\right), \text { where } \\
l_{k / N} & :=\frac{d^{2}}{d r^{2}}-\frac{(k / N)^{2}-1 / 4}{r^{2}}
\end{aligned}
$$

(It is understood that $W$ acts on the radial part of the decomposition (4.35).)

Note that $l_{\nu}$ in (4.39) is the Bessel differential operator of order $\nu$ acting on $L^{2}\left(\mathbb{R}_{+}, d r\right)$, where $d r$ denotes the Lebesgue measure. It is known that $\left.l_{\nu}\right|_{C_{c}^{\infty}\left(\mathbb{R}_{+}\right)}$is essentially selfadjoint iff $|\nu| \geq 1$. See, for example, [AG93]. 
Proposition 4.17. Let $M$ be the $N$-covering surface of $\mathbb{R}^{2} \backslash\{(0,0)\}, N<\infty$. Let $L$ be the Nelson-Laplace operator in (4.14), and $\mathscr{D}_{1}$ be the deficiency space in (4.16). Then $\mathscr{D}_{1}$ is the linear span of the following functions:

$$
K_{k / N}(r) e^{ \pm i \theta(k / N)}
$$

where $k=0, \ldots, N-1$; and $K_{\nu}$ denotes the modified Bessel function of the second kind of order $\nu$. In particular, $L$ has deficiency indices $(2 N-1,2 N-1)$.

Proof. Let $\psi \in \mathscr{D}_{1}$, i.e., $\psi$ is the solution to the following equation:

$$
\Delta \psi=\psi, \psi \in C^{\infty}(M) \cap L^{2}(M) ;
$$

see (4.16). Using (4.35), we may write

$$
\begin{aligned}
\psi(r, \theta) & =\sum_{k \in \mathbb{Z}} \widehat{\psi}_{k / N}(r) e^{i k \theta / N}, \text { where } \\
\widehat{\psi}_{k / N}(r) & :=\frac{1}{2 \pi N} \int_{0}^{2 \pi N} \psi(r, \theta) e^{-i k \theta / N} d \theta,
\end{aligned}
$$

so that

$$
\begin{aligned}
\Delta \psi & =\sum_{k \in \mathbb{Z}} \Delta\left(\sum_{k \in \mathbb{Z}} \widehat{\psi}_{k / N}(r) e^{i k \theta / N}\right) \\
& =\sum_{k \in \mathbb{Z}}\left(\left(\frac{1}{r} \frac{d}{d r}\left(r \frac{d}{d r}\right)-\frac{(k / N)^{2}}{r^{2}}\right) \widehat{\psi}_{k / N}(r)\right) e^{i k \theta / N} .
\end{aligned}
$$

It follows that $\Delta \psi=\psi$ iff

$$
\left(\frac{1}{r} \frac{d}{d r}\left(r \frac{d}{d r}\right)-\frac{(k / N)^{2}}{r^{2}}\right) \widehat{\psi}_{k / N}(r)=\widehat{\psi}_{k / N}(r) .
$$

By [AG93] and the discussion above, the only solution to (4.42) in $L^{2}\left(\mathbb{R}_{+}, r d r\right)$ is a scalar multiple of $K_{k / N}(r)$, for $|k / N|<1$, i.e.,

$$
\widehat{\psi}_{k / N}(r)=K_{k / N}(r), k=0, \ldots, N-1 .
$$

Therefore, by (4.40), we have

$$
\psi(r, \theta)=\sum_{k \in \mathbb{Z}} \widehat{\psi}_{k / N}(r) e^{i k \theta / N}=\sum_{k \in \mathbb{Z}} K_{k / N}(r) e^{i k \theta / N}
$$

for $k=0, \ldots, N-1$; which is the assertion.

Acknowledgement. The co-authors thank the following colleagues for helpful and enlightening discussions: Professors Daniel Alpay, Sergii Bezuglyi, Paul Muhly, MyungSin Song, Wayne Polyzou, Gestur Olafsson, Keri Kornelson, and members in the Math Physics seminar at the University of Iowa. 


\section{REFERENCES}

[AG93] N. I. Akhiezer and I. M. Glazman, Theory of linear operators in Hilbert space, Dover Publications Inc., New York, 1993, Translated from the Russian and with a preface by Merlynd Nestell, Reprint of the 1961 and 1963 translations, Two volumes bound as one. MR 1255973 (94i:47001)

[AJSV13] Daniel Alpay, Palle Jorgensen, Ron Seager, and Dan Volok, On discrete analytic functions: products, rational functions and reproducing kernels, J. Appl. Math. Comput. 41 (2013), no. 1-2, 393-426. MR 3017129

[Arn78] D. Arnal, Analytic vectors and irreducible representations of nilpotent Lie groups and algebras, Lett. Math. Phys. 2 (1977/78), no. 6, 429-434. MR 513110 (80i:22024)

[Ben02] Djilali Benayat, Local representations of Lie groups, Far East J. Math. Sci. (FJMS) 4 (2002), no. 3, 389-403. MR 1902945 (2003i:20093)

[BGJR88] Ola Bratteli, Frederick M. Goodman, Palle E. T. Jorgensen, and Derek W. Robinson, The heat semigroup and integrability of Lie algebras, J. Funct. Anal. 79 (1988), no. 2, 351-397. MR 953908 (90a:47105)

[Che72] Paul R. Chernoff, Some remarks on quasi-analytic vectors, Trans. Amer. Math. Soc. 167 (1972), 105-113. MR 0295125 (45 \#4193)

[CJ12] Ilwoo Cho and Palle E. T. Jorgensen, Operators induced by graphs, Lett. Math. Phys. 102 (2012), no. 3, 323-369. MR 2989487

[dG83] J. de Graaf, A theory of generalized functions based on holomorphic semigroups. I. Introduction and survey, Nederl. Akad. Wetensch. Indag. Math. 45 (1983), no. 4, 407-420. MR 731824 (85i:46051)

[dG84]_ A theory of generalized functions based on holomorphic semigroups. III. Linear mappings, tensor products and kernel theorems, Nederl. Akad. Wetensch. Indag. Math. 46 (1984), no. 2, 173-187. MR 749530 (86b:46060b)

[DS88] Nelson Dunford and Jacob T. Schwartz, Linear operators. Part II, Wiley Classics Library, John Wiley \& Sons, Inc., New York, 1988, Spectral theory. Selfadjoint operators in Hilbert space, With the assistance of William G. Bade and Robert G. Bartle, Reprint of the 1963 original, A Wiley-Interscience Publication. MR 1009163 (90g:47001b)

[Frö80] J. Fröhlich, Unbounded, symmetric semigroups on a separable Hilbert space are essentially selfadjoint, Adv. in Appl. Math. 1 (1980), no. 3, 237-256. MR 603131 (82c:47044)

[FS66] M. Flato and D. Sternheimer, Local representations and mass spectrum, Phys. Rev. Lett. 16 (1966), 1185-1186. MR 0226913 (37 \#2499)

[GKS11] Heiko Gimperlein, Bernhard Krötz, and Henrik Schlichtkrull, Analytic representation theory of Lie groups: general theory and analytic globalizations of Harish-Chandra modules, Compos. Math. 147 (2011), no. 5, 1581-1607. MR 2834734 (2012i:22017)

[GT12] Rahul Garg and Sundaram Thangavelu, On the structure of analytic vectors for the Schrödinger representation, Monatsh. Math. 167 (2012), no. 1, 61-80. MR 2942964

[JM84] Palle E. T. Jorgensen and Robert T. Moore, Operator commutation relations, Mathematics and its Applications, D. Reidel Publishing Co., Dordrecht, 1984, Commutation relations for operators, semigroups, and resolvents with applications to mathematical physics and representations of Lie groups. MR 746138 (86i:22006)

[Jør76] Palle T. Jørgensen, Perturbation and analytic continuation of group representations, Bull. Amer. Math. Soc. 82 (1976), no. 6, 921-924. MR 0476922 (57 \#16473)

[Jor86] Palle E. T. Jorgensen, Analytic continuation of local representations of Lie groups, Pacific J. Math. 125 (1986), no. 2, 397-408. MR 863534 (88m:22030)

[Jor87] _ Analytic continuation of local representations of symmetric spaces, J. Funct. Anal. 70 (1987), no. 2, 304-322. MR 874059 (88d:22021) 
[JPT13] Palle E. T. Jorgensen, Steen Pedersen, and Feng Tian, Momentum operators in two intervals: spectra and phase transition, Complex Anal. Oper. Theory 7 (2013), no. 6, 1735-1773. MR 3129890

[JPT14] Palle Jorgensen, Steen Pedersen, and Feng Tian, Restrictions and extensions of semibounded operators, Complex Anal. Oper. Theory 8 (2014), no. 3, 591-663. MR 3167762

[LP89] Peter D. Lax and Ralph S. Phillips, Scattering theory, second ed., Pure and Applied Mathematics, vol. 26, Academic Press, Inc., Boston, MA, 1989, With appendices by Cathleen S. Morawetz and Georg Schmidt. MR 1037774 (90k:35005)

[Nee06] Karl-Hermann Neeb, Towards a Lie theory of locally convex groups, Jpn. J. Math. 1 (2006), no. 2, 291-468. MR 2261066 (2007k:22020)

[Nee11] Karl-H. Neeb, On analytic vectors for unitary representations of infinite dimensional Lie groups, Ann. Inst. Fourier (Grenoble) 61 (2011), no. 5, 1839-1874 (2012). MR 2961842

[Nel59] Edward Nelson, Analytic vectors, Ann. of Math. (2) 70 (1959), 572-615. MR 0107176 (21 \#5901)

[Pra91] Humberto E. Prado, A geometric construction of local representations of local Lie groups, Acta Appl. Math. 25 (1991), no. 1, 87-98. MR 1140759 (92j:22014)

[Rob89] Derek W. Robinson, Lipschitz operators, J. Funct. Anal. 85 (1989), no. 1, $179-211$. MR 1005861 (90j:22011)

[Rob90] _ The heat semigroup and integrability of Lie algebras: Lipschitz spaces and smoothness properties, Comm. Math. Phys. 132 (1990), no. 1, 217-243. MR 1069210 (91i:17012)

[Rus87] Jan Rusinek, Analytic vectors and integrability of Lie algebra representations, J. Funct. Anal. 74 (1987), no. 1, 10-23. MR 901228 (88i:22041)

[Sch84] Konrad Schmüdgen, On commuting unbounded selfadjoint operators. I, Acta Sci. Math. (Szeged) 47 (1984), no. 1-2, 131-146. MR 755571 (86b:47045)

[Sch85] _ On commuting unbounded selfadjoint operators. III, Manuscripta Math. 54 (1985), no. 1-2, 221-247. MR 808690 (87h:47061)

[Sch86a]__ A note on commuting unbounded selfadjoint operators affiliated to properly infinite von Neumann algebras. II, Bull. London Math. Soc. 18 (1986), no. 3, 287-292. MR 829589 (87g:47079)

[Sch86b] On commuting unbounded selfadjoint operators. IV, Math. Nachr. 125 (1986), 83-102. MR 847352 (88j:47026)

[Seg64] I. E. Segal, Infinite-dimensional irreducible representations of compact semi-simple groups, Bull. Amer. Math. Soc. 70 (1964), 155-160. MR 0158028 (28 \#1256)

[SF84] K. Schmüdgen and J. Friedrich, On commuting unbounded selfadjoint operators. II, Integral Equations Operator Theory 7 (1984), no. 6, 815-867. MR 774726 (86i:47032)

[SW71] Elias M. Stein and Guido Weiss, Introduction to Fourier analysis on Euclidean spaces, Princeton University Press, Princeton, N.J., 1971, Princeton Mathematical Series, No. 32. MR 0304972 (46 \#4102)

[Tia11] Feng Tian, On commutativity of unbounded operators in Hilbert space, Ph.D. thesis, University of Iowa, May 2011.

[vN30] J. v. Neumann, Allgemeine Eigenwerttheorie Hermitescher Funktionaloperatoren, Math. Ann. 102 (1930), no. 1, 49-131. MR 1512569

[Voh72] U. C. Vohra, On almost analytic vectors in a strict sense, C. R. Acad. Bulgare Sci. 25 (1972), 889-891. MR 0319105 (47 \#7651)

[Wat44] G. N. Watson, A Treatise on the Theory of Bessel Functions, Cambridge University Press, Cambridge, England, 1944. MR MR0010746 (6,64a) 
(Palle E.T. Jorgensen) Department of Mathematics, The University of Iowa, Iowa City, IA 52242-1419, U.S.A.

E-mail address: palle-jorgensen@uiowa.edu

$U R L:$ http://www.math.uiowa.edu/〜jorgen/

(Feng Tian) Department of Mathematics, Wright State University, Dayton, OH 45435, U.S.A.

E-mail address: feng.tian@wright.edu

$U R L:$ http: //www. wright.edu/ feng.tian/ 